\title{
Instrumentation Progress at the Giant Magellan Telescope Project
}

\author{
George H. Jacoby $^{* a}$, R. Bernstein ${ }^{\mathrm{a}}$, A. Bouchez ${ }^{\mathrm{a}}$, M. Colless ${ }^{\mathrm{b}}, \mathrm{J}_{\text {. Crane }}^{\mathrm{c}}$, D. DePoy ${ }^{\mathrm{d}}$, B. Espeland ${ }^{\mathrm{b}}, \mathrm{T}$. \\ Hare $^{\text {c }}$, D. Jaffe ${ }^{\mathrm{e}}$, J. Lawrence, J. Marshall ${ }^{\mathrm{d}}$, P. McGregor ${ }^{\mathrm{b}}$, S. Shectman ${ }^{\mathrm{c}}$, R. Sharp ${ }^{\mathrm{b}}$, A. \\ Szentgyorgyi $^{\mathrm{g}}$, A. Uomoto ${ }^{\mathrm{c}}, \mathrm{B}$. Walls ${ }^{\mathrm{a}}$ \\ ${ }^{a}$ Giant Magellan Telescope Organization, 465 N. Halstead St., Pasadena, CA 91107 \\ ${ }^{b}$ Research School of Astronomy and Astrophysics, The Australian National University, Cotter Road, \\ Weston, ACT, 2611, Australia \\ ${ }^{\mathrm{C} C a r n e g i e}$ Observatories, 813 Santa Barbara St., Pasadena, CA 91101 \\ ${ }^{\mathrm{d}}$ Department of Physics and Astronomy, Texas A\&M University, College Station, TX 77843 \\ ${ }^{\mathrm{e}}$ Department of Astronomy, University of Texas at Austin, Austin, TX \\ ${ }^{\mathrm{f}}$ Australian Astronomical Observatory, PO Box 296 Epping, NSW 1710, Australia \\ ${ }^{\mathrm{g}}$ Harvard-Smithsonian Center for Astrophysics, 60 Garden St., Cambridge, MA 0213891101
}

\begin{abstract}
Instrument development for the $24 \mathrm{~m}$ Giant Magellan Telescope (GMT) is described: current activities, progress, status, and schedule. One instrument team has completed its preliminary design and is currently beginning its final design (GCLEF, an optical 350-950 nm, high-resolution and precision radial velocity echelle spectrograph). A second instrument team is in its conceptual design phase (GMACS, an optical 350-950 nm, medium resolution, 6-10 arcmin field, multiobject spectrograph). A third instrument team is midway through its preliminary design phase (GMTIFS, a near-IR YJHK diffraction-limited imager/integral-field-spectrograph), focused on risk reduction prototyping and design optimization. A fourth instrument team is currently fabricating the 5 silicon immersion gratings needed to begin its preliminary design phase (GMTNIRS, a simultaneous JHKLM high-resolution, AO-fed, echelle spectrograph). And, another instrument team is focusing on technical development and prototyping (MANIFEST, a facility robotic, multifiber-feed, with a 20 arcmin field of view). In addition, a medium-field ( 6 arcmin, 0.06 arcsec/pix) optical imager will support telescope and AO commissioning activities, and will excel at narrow-band imaging. In the spirit of advancing synergies with other groups, the challenges of running an ELT instrument program and opportunities for cross-ELT collaborations are discussed.
\end{abstract}

Keywords: GMT, ELT, spectrograph, integral-field spectrograph, multi-object spectrograph, optical instruments, infrared instruments

\section{INTRODUCTION}

The GMT instrumentation program has followed the usual progression for instrument development during the past 10 years, beginning with the run-up to the GMT Conceptual Design Review ${ }^{1}$ in 2006 . That process was described in our previous SPIE report ${ }^{2}$. Briefly, early concepts were discussed within the GMT community, followed by conceptual designs with a subsequent down-select to define a first generation suite, and then preliminary designs for those few selected instruments. The status of the GMT instrument developments currently spans that entire range of process and beyond, from early concept to final design.

In this paper, we present an overview of those developments for the first generation instrument suite. Details of the individual instrument designs and their status can be found elsewhere in these procedings ${ }^{3,4,5,6,7}$. We also describe a modest optical imager that recently has been added to the first generation of instruments, as well as several early ideas

*gjacoby@gmto.org; +1-520-904-4135

Ground-based and Airborne Instrumentation for Astronomy VI, edited by Christopher J. Evans, Luc Simard, Hideki Takami Proc. of SPIE Vol. 9908, 99081U · (c) 2016 SPIE · CCC code: 0277-786X/16/\$18 · doi: 10.1117/12.2232809 
for future instruments. The timeline for developing ELT-class instruments is so extended ( $>10$ years) that all large telescope projects have started down the future generation path.

In our final sections, we complement the look forward to completion of the first generation with a retrospective view, briefly discussing the challenges faced by all ELT instrument teams, and finally, describe how some of those challenges may be mitigated in the future.

\section{THE GMT PROJECT STATUS}

The GMT project-wide status is described in detail by McCarthy ${ }^{8}$ elsewhere in this conference. Here, we include a short discussion of those elements of the greater GMT project that impact instrument development most directly.

\subsection{Requirements}

Instrument designs are relatively static compared to the rapidity of scientific discovery. The tools we proposed 10 years ago to solve problems of the past decade may not be the same tools you would choose today. To mitigate that concern, telescope projects must revisit their science cases from time to time in order to assess whether tomorrow's astronomical questions will impact today's requirements. We can then ask ourselves if the instruments we are building are the right ones. Not surprisingly, first generation ELT instruments have very broad application because they were chosen with breadth as a criterion. But, we need to be careful not to allow inertia to cloud our judgement on those early decisions.

GMTO revised its science book in $2012^{10}$, and it may be time for another update given recent discoveries (e.g., the vast collection of known exoplanets, discovery of gravitational waves ${ }^{9}$ ). Science requirements flow from these science drivers, and lower level requirements flow from those. GMTO is in the process of revising and reviewing its top level requirements for the final time before releasing purchase tenders for major procurements (e.g., telescope mount, facility enclosure) in order to ensure a tight connection back to the science.

Clearly, instruments may be impacted by the flow down of changes from higher level requirements.

\subsection{Telescope Design}

In response to the GMT Preliminary Design Review in January 2014, several aspects of the telescope structure have been carefully scrutinized. Key concerns have been addressed, including the integral nature of the 7 mirror cells to the telescope structure, the potential for large amplification of seismic accelerations (up to $5 \mathrm{~g}$ ), and the installation, number of ports, and mounting of instruments.

All of these impact the instruments, and the latter, very directly.

\subsection{Primary Mirrors}

Four of the 8 mirrors have been cast, including the central segment. They are in various stages of fabrication. Mirror 5 is scheduled to be cast within the next year and glass for mirror 6 is being purchased with deliveries scheduled for the end of 2016.

The impact of mirror delivery on the instruments should be minor, but there are two aspects to consider. First, the mirror segments are close to the critical path and may drive the overall project schedule. For instrument development, this could imply a work slowdown (or more optimistically, a speedup). Second, GMT plans to begin operation with a subset of the seven mirrors perhaps four or five, and so, IR instruments will have to swap out their cold pupil stop to match the primary mirror configuration as additional segments are delivered and installed.

\subsection{Adaptive Optics (AO)}

GMT has adopted a staged implementation of capabilities (see section 9 of $\mathrm{McCarthy}^{8}$ ) in which the AO systems are deferred to the out-years of the project. Because the GMT IR instruments (GMTIFS and GMTNIRS, in particular) require $\mathrm{AO}$, the instrument suite will be restricted to natural seeing operation with visible wavelength instruments. Strategies are being developed to bring a natural seeing IR capability to GMT close to first light (see Section 4.1). Furthermore, GMT's GLAO implementation is a key mode of the AO system that is also deferred, thereby impacting the performance of the natural seeing instruments as well. 


\subsection{Enclosure}

Normally, the enclosure doesn't interact strongly with instrument development. GMT's enclosure design is undergoing several trade studies ${ }^{11,12}$ that will flow to considerations regarding instrument handling, installation, and maintenance. While these are minor concerns, they do impact aspects of the design.

\subsection{Schedule}

As noted in Section 2.3, the negotiated date for instrument delivery derives from the schedule. If the date is pushed out, there are cost implications along with other risks associated with delays (e.g., loss of key personal, procurement costs, vendor loss). The GMT schedule that is shown in Figure 1 is purely technically driven and assumes that funding is available when needed. GMT could go into operation with four primary mirror segments in late 2022 , but the full array of segments requires another few years.

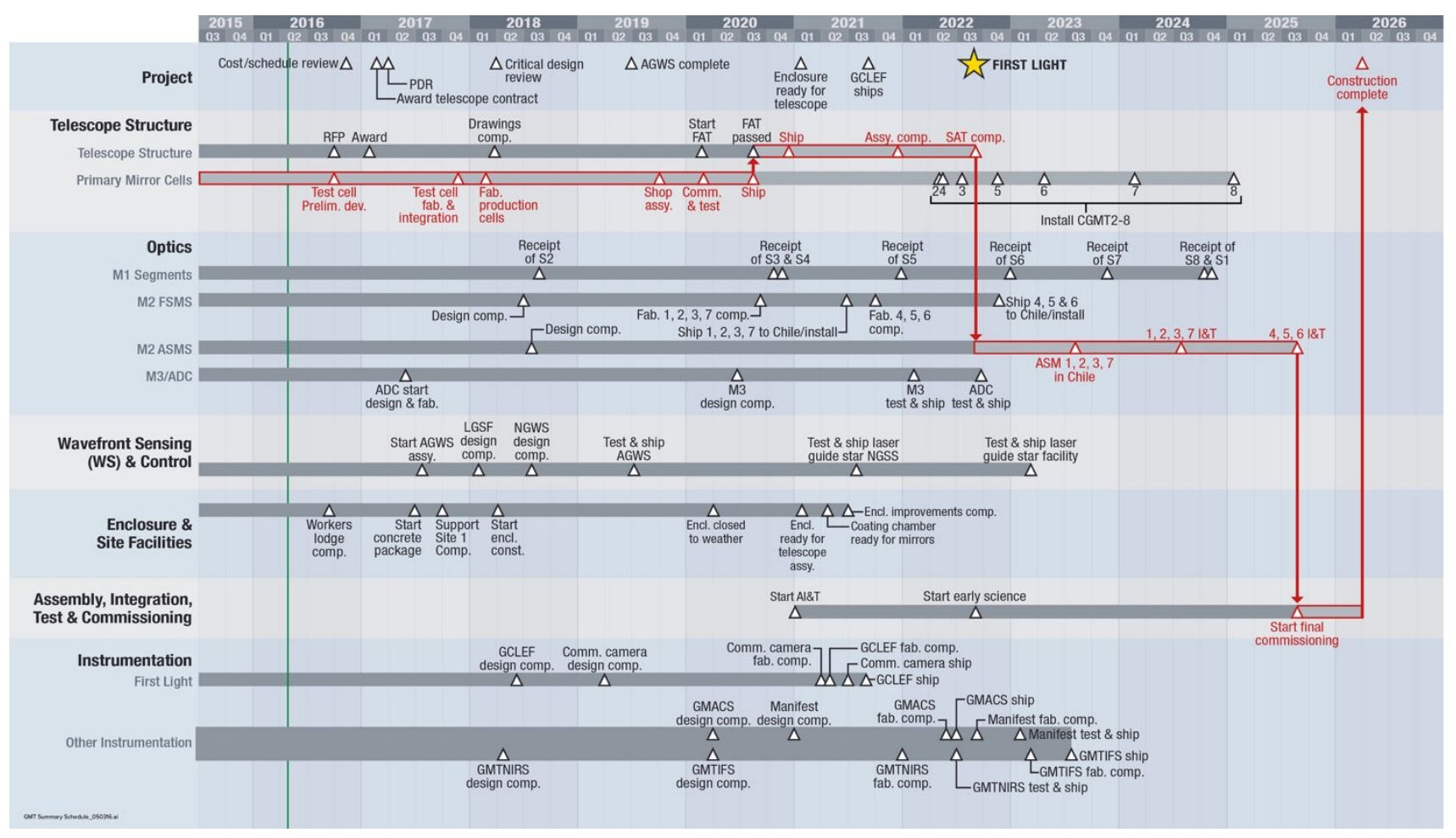

Figure 1. Summary schedule for the GMT project. The 2 grey bars along the bottom represent the instrument schedules.

\section{FIRST GENERATION INSTRUMENT SUITE}

Table 1 and Table 2 summarize the instrument capabilities in GMT's first generation suite. These are color-coded by the status of their development stage. With the exception of ComCam, a new addition to the family, the other five instruments are described in far more detail in other papers at this conference. They also have been discussed at previous conferences in this series. Thus, the instrument descriptions we present here are brief. 
Table 1. The 6 instruments currently being supported as part of the GMT first generation collection. Instrument names are in bold, and their observing modes are NS=natural seeing, GLAO=ground layer adaptive optics, LTAO=laser tomography adaptive optics, or NGSAO=natural guide star adaptive optics. The development status is defined by the color codes shown under Capabilities description and defined in Table 2. There is no spectral Resolution given for MANIFEST because it is not truly an instrument but rather an instrument feed, where the resolution is defined by the client instrument.

\begin{tabular}{|c|c|c|c|c|}
\hline Instrument / Mode & Capabilities & $\begin{array}{c}\lambda \text { Range, } \\
\mu \mathrm{m}\end{array}$ & Resolution & Field of View \\
\hline $\begin{array}{l}\text { G-CLEF / NS, GLAO, } \\
\text { NGSAO }\end{array}$ & $\begin{array}{l}\text { Optical High Resolution } \\
\text { Spectrograph / PRV }\end{array}$ & $0.35-0.95$ & $19,000-108,000$ & $7 \times 0.7,1.2$ " fibers \\
\hline $\begin{array}{l}\text { GMTIFS / LTAO, } \\
\text { NGSAO }\end{array}$ & NIR AO-fed IFS / Imager & $0.95-2.5$ & $5,000 \& 10,000$ & $10 / 400 \operatorname{arcsec}^{2}$ \\
\hline GMACS / NS, GLAO & $\begin{array}{l}\text { Wide-Field Optical Multi- } \\
\text { Object Spectrograph }\end{array}$ & $0.35-0.95$ & $\begin{array}{c}1,000-6,000 \\
(8 \mathrm{~K} \text { with MANIFEST) }\end{array}$ & 7.5' diameter \\
\hline ComCam / NS, GLAO & Optical Imager & $0.34-1.0$ & $0.06 \mathrm{arcsec} / \mathrm{pix}$ & $6 \times 6$ arcmin \\
\hline $\begin{array}{l}\text { GMTNIRS / NGSAO, } \\
\text { LTAO }\end{array}$ & $\begin{array}{l}\text { JHKLM AO-fed High } \\
\text { Resolution Spectrograph }\end{array}$ & $1.1-5.3$ & $\begin{array}{c}50,000 / 75,000 \\
(\mathrm{JHK} / \mathrm{LM})\end{array}$ & 1.2" long-slit \\
\hline MANIFEST / NS, GLAO & Facility Robotic Fiber Feed & $0.36-1.0$ & & 20 ' diameter \\
\hline
\end{tabular}

Table 2. The legend defining the instrument development stages, its current activity, and next planned step, following the the color codes shown under "Capabilities" in Table 1.

\begin{tabular}{|l|l|}
\hline Current Phase & Next Phase \\
\hline Final Design & Fabrication \\
\hline Preliminary Design & Final Design \\
\hline Conceptual Design & Preliminary Design \\
\hline Silicon Grating Technology Development & Preliminary Design \\
\hline Science demonstrator closeout & Concept Design \\
\hline
\end{tabular}

\subsection{G-CLEF}

The GMT - CfA Large Earth Finder (G-CLEF) is a general purpose visible echelle spectrograph $(3500-9500 \AA)$ that also provides precision radial velocity (PRV) capabilities $(<50 \mathrm{~cm} / \mathrm{s}$ per observation; goal $<10 \mathrm{~cm} / \mathrm{s}$ with multiple observations). G-CLEF will reside in a gravity invariant and temperature controlled environment on the azimuth disk of the telescope. An optical and fiber relay system will transfer light from the telescope to G-CLEF and will scramble the light within each of the seven sub-apertures in the process. The instrument will support several observing modes (PRV, high throughput, medium resolution, and multi-object over $300 \operatorname{arcmin}^{2}$ when fed by MANIFEST) with a range of spectral resolutions $(19,000,35,000,108,000)$ and input aperture sizes.

The G-CLEF ${ }^{3}$ project is being led by Andrew Szentgyorgyi (SAO) with support from GMT partners at Carnegie Observatories and Korea Astronomy and Space Science Institute (KASI). The targeted science includes:

- Exoplanets - precise velocities; atmospheric properties

- Stars - abundances, isotopes, and velocities

- Dwarf galaxies - abundances; dark matter

- Cosmology - chemical evolution; fine structure constant 


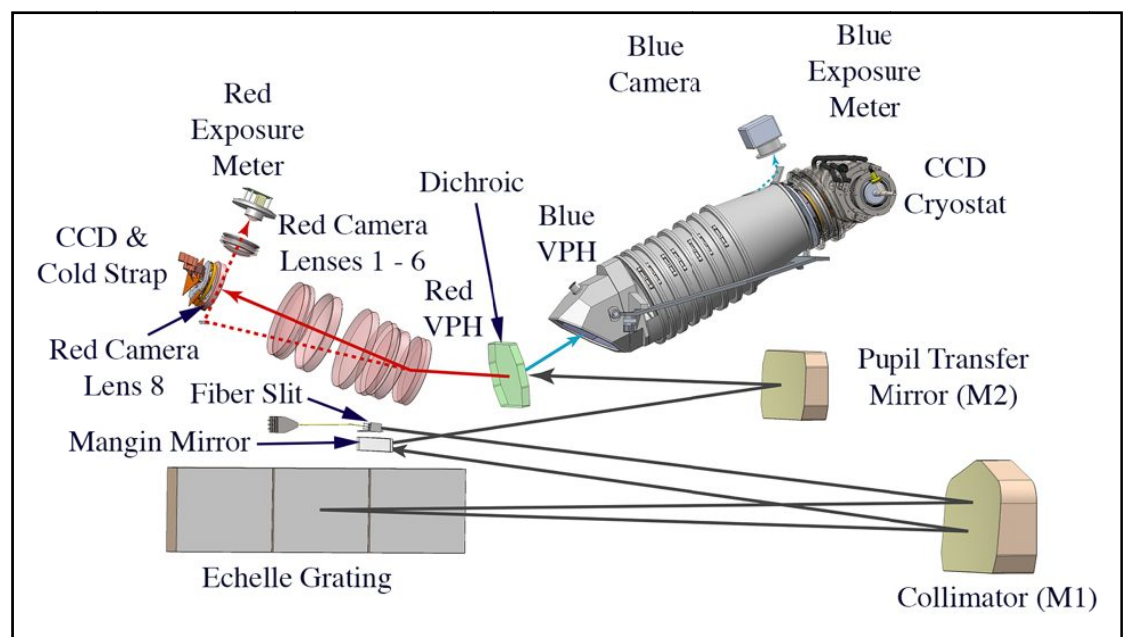

Figure 2. The optical layout for G-CLEF

The G-CLEF team successfully passed its preliminary design review in October 2015 and has begun its final design study. It will be the first major science instrument for GMT (see Figure 1) and so it follows a rapid development and delivery schedule.

\subsection{GMTIFS}

The GMT Integral Field Spectrograph (GMTIFS) builds on the legacy of the NIFS instrument on Gemini. It uses an image slicing mirror system to reformat the focal plane onto the detector. GMTIFS is an AO-fed spectrograph and thus, requires that the GMT adaptive secondary mirrors be operational, scheduled for mid-2024.

Spaxel scales of 6,12, 25, and 50 mas are provided to sample the J-band diffraction limit at the small end, and to enable low surface brightness sensitivity at the large end. The spectral resolution of 5,000 allows for full spectral coverage in any one of the $\mathrm{Y}, \mathrm{J}, \mathrm{H}$, or K-bands in a single setting. A higher resolution grating $(\mathrm{R}=10,000)$ provides good kinematic resolution of small and distant galaxies undergoing assembly. The instrument also has an imaging channel that will cover the laser tomography AO (LTAO) field (20.4") while critically sampling the PSF in the J-band with its 5 mas pixels.

The GMTIFS project ${ }^{4}$ had been led by Peter McGregor (ANU) until his unfortunate and untimely passing last year. Currently, Rob Sharp (ANU) is leading the project. As a general purpose instrument, the scope of GMTIFS science is extensive; a few sample areas include:

- Young stars - star and planet formation processes

- Nearby galaxies - chemical enrichment history

- First galaxies - structure and assembly

- Massive black holes - masses and physics of galaxy nuclei

- Gamma ray bursts - intergalactic medium at $\mathrm{z} \sim 7$ 

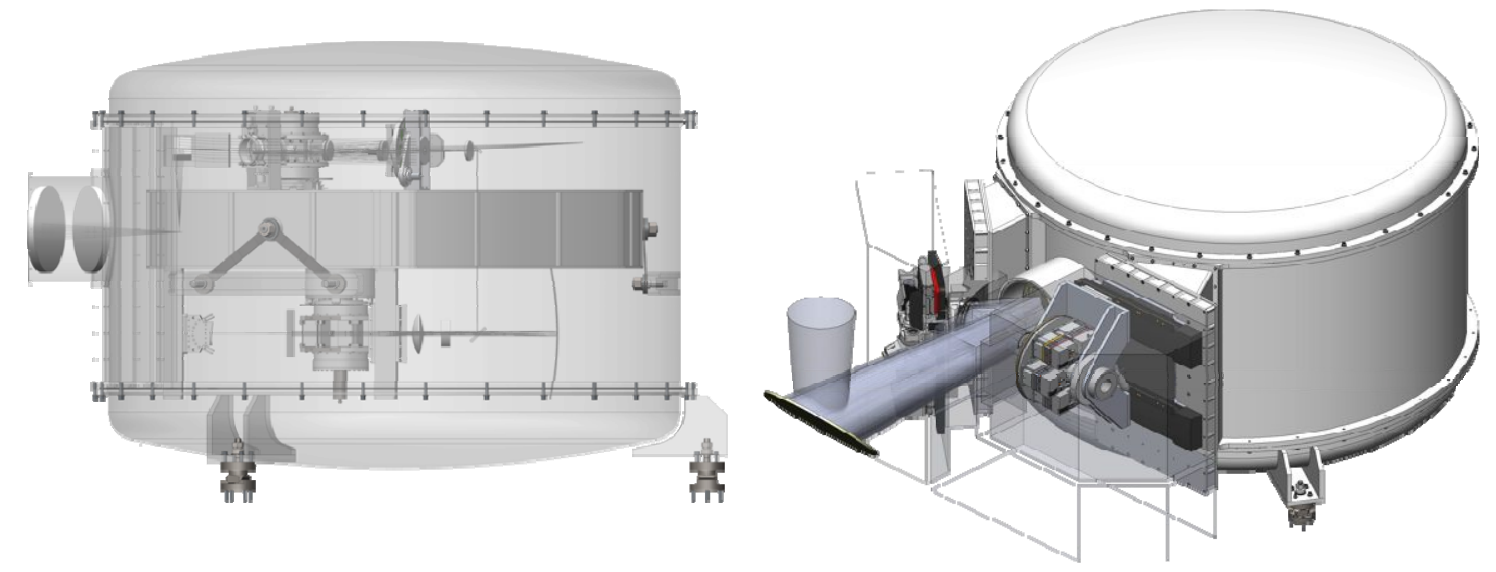

Figure 3. The GMTIFS layout: Left - partially populated optical table within the cryostat volume. Final design for all optical elements is complete with some mechanical mounts awaiting tolerancing. The central chamber of the cold work surface houses the OIWFS beam steering mirror, while the fore optics, imager, and IFS plus on-instrument wavefront sensor (OIWFS) are distributed across the upper and lower zones. Right - A view of the GMTIFS cryostat showing the GMT tertiary mirror folding the 180 arcsec diameter field to GMTIFS. The dichroic cryostat window reflects $\lambda<0.95 \mu \mathrm{m}$ to the external NGSAO and LTAO wavefront sensors mounted to the front of the cryostat.

The GMTIFS team is about $70 \%$ through its preliminary design study. The focus of effort has been to retire risk developments, including precision (i.e., sub-micron) cryogenic control of the steering mirror that directs the AO tip/tilt guide star to the wavefront sensor, optical design for the spectrograph and ADC, and wavefront sensor detector systems. Related activities include cold operation of the wavefront sensor deformable mirror for off-axis guide stars and exploration of eAPD arrays for the wavefront sensor. It will be the first AO imager instrument for GMT (see Figure 1) and is scheduled for delivery in mid-2023.

\subsection{GMACS}

The GMT Multi-object Astronomical and Cosmological Spectrograph (GMACS) is a general purpose, visible light (3500-9500 §̊), multi-object, slit spectrograph (MOS) that covers a relatively wide field (approximately 7.5' diameter). Spectral resolutions of 1000-6000 are enabled by multiple VPH gratings in each of the red and blue channels. The current GMACS design also includes an imaging mode with a scale of $0.05 \% /$ pix.

The GMACS 5 team is led by Darren DePoy (Texas A\&M Univ) with support from GMT's South Korean partners at Kyung Hee University and The São Paulo Research Foundation. As an optical MOS, GMACS has extremely wide application across astronomy. Some of its key science areas include:

- Stellar evolution - brown dwarfs; white dwarfs

- Nearby galaxies - chemical enrichment history; dark matter distribution

- Distant galaxies - Ly $\alpha$ luminosity function at $\mathrm{z}<6.5$; chemical enrichment of the ISM

- $\quad$ LSST - follow-up of transients; redshift surveys 


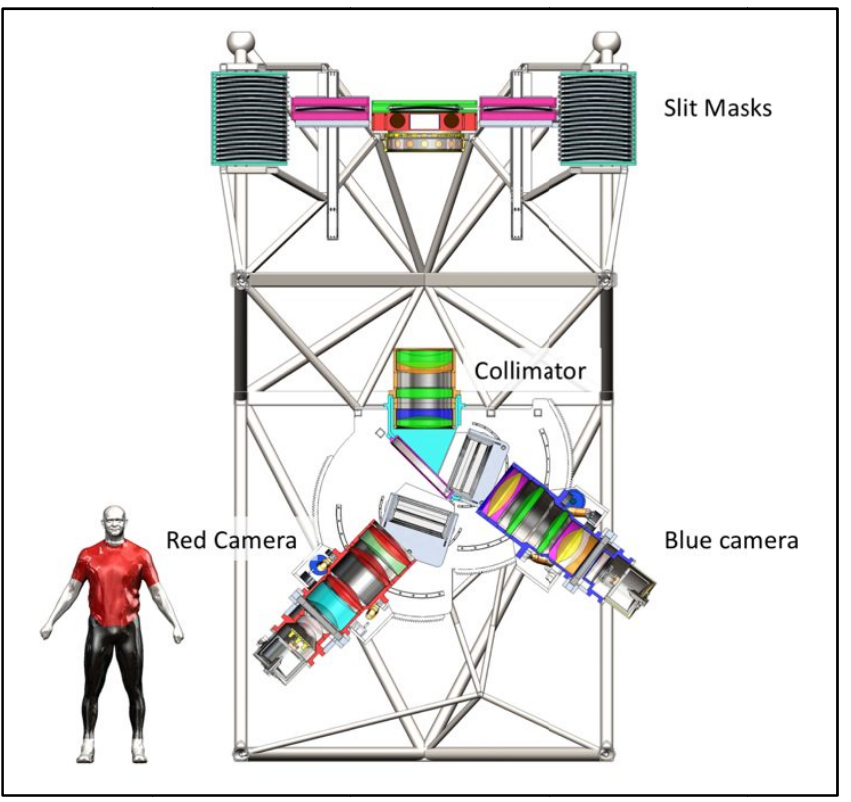

Figure 4. A simplified layout for GMACS in one of the four direct Gregorian instrument bays.

The GMACS team is beginning a renewed conceptual design for the instrument with a reduced field of view compared to the 2011 concept. That design change was driven primarily by funding limitations. Independently, a requirement for a partial J-band channel was removed after exploring the technical risks and costs relative to the science return for the modest near-IR capability. GMT's intent is for GMACS to be the second major science instrument, arriving about 8 months after G-CLEF.

\subsection{GMTNIRS}

The GMT Near-IR spectrometer (GMTNIRS) is a $1.1-5.3 \mu \mathrm{m}$ echelle, recording the full JHKLM bands simultaneously on five spectrograph modules. It uses silicon immersion gratings to achieve high spectral resolution in a compact format. The fixed slit width of $\sim 65$ mas is well matched to the diffraction-limited image size from a single $8.4 \mathrm{~m}$ GMT primary mirror segment. At that slit width, GMTNIRS will deliver spectral resolution of $\sim 50,000$ in JHK and 75,000 in LM.

GMTNIRS is led by Dan Jaffe (Univ of Texas Austin) with collaboration from GMT's South Korean partner KASI. While it is optimized for studies of young stellar objects, debris disks, and protoplanetary systems, GMTNIRS has much wider application. Recent results from its forerunner, IGRINS ${ }^{14}$, demonstrates the scientific extent to which a high resolution IR echelle spectrograph can be applied. Here are some areas where GMTNIRS will play a scientific role:

- Exoplanets - atmospheric chemistry; internal structure

- Stars - formation processes of stars, disks, and planets

- Galaxy - chemical composition of stars and nebulae

- Nearby galaxies - chemical evolution history

- Distant galaxies - composition of the universe in Lya systems 


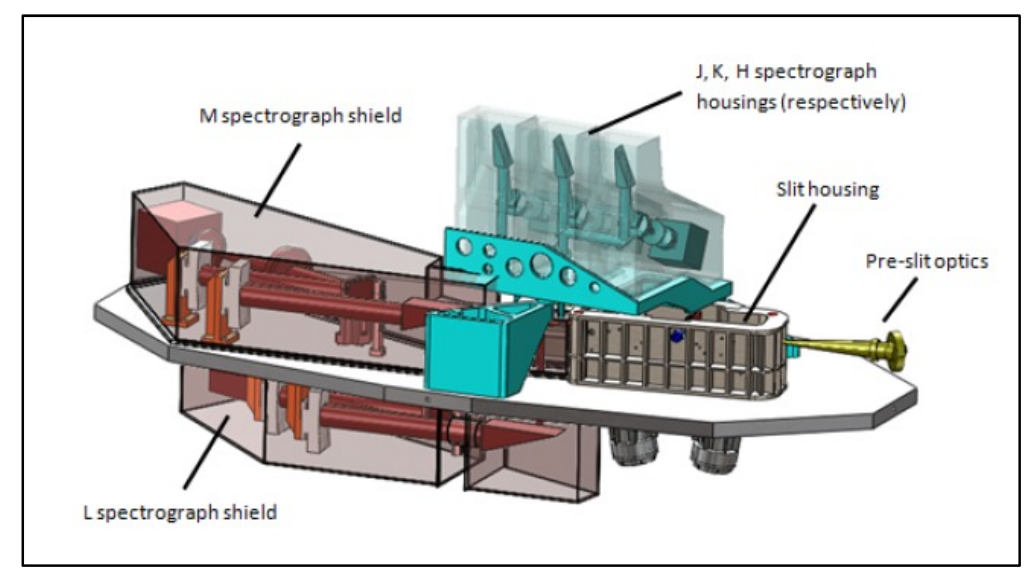

Figure 5. A simplified layout of the GMTNIRS spectrograph. The JHK modules are nearly identical, as are the L and M modules.

The GMTNIRS team is developing the techniques to fabricate the unique silicon immersion gratings that the instrument requires. Several of the smaller $(100 \mathrm{~mm})$ JHK gratings have been completed through the difficult stages and results look excellent (Figure 6, left).
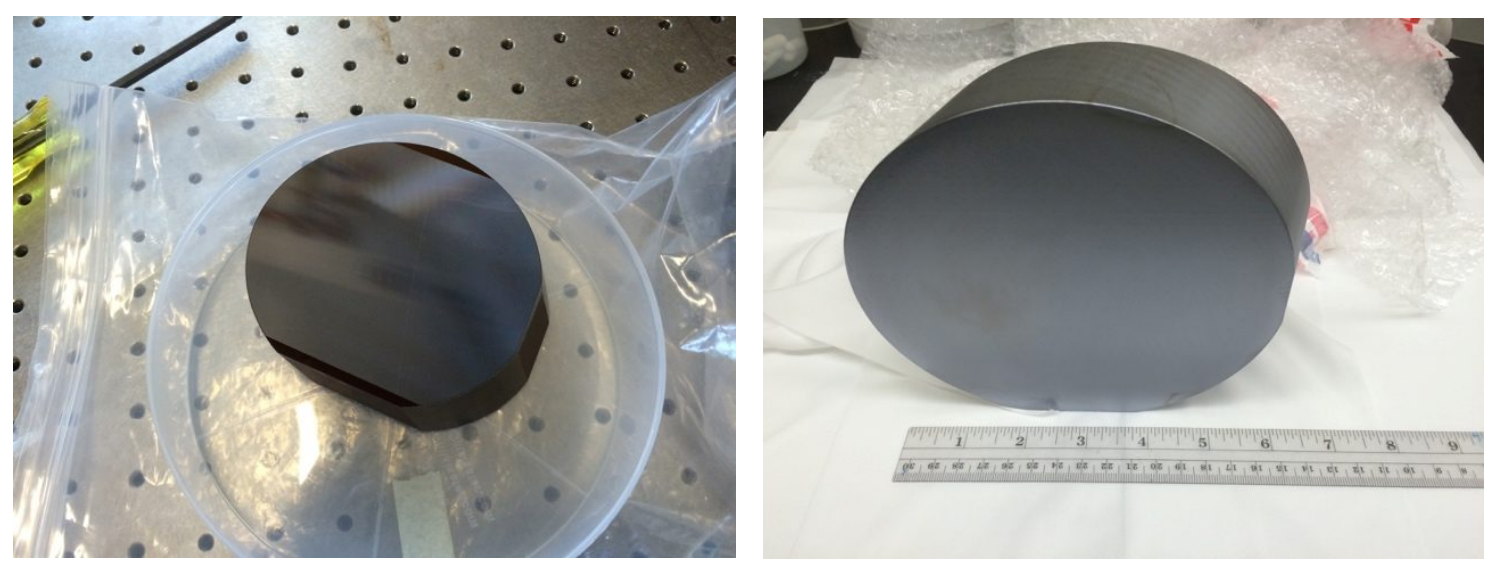

Figure 6. A near-final processed K-band grating for GMTNIRS (left) and a substrate for L-band that has been x-ray aligned and cut.

The larger $(150 \mathrm{~mm}) \mathrm{L}$ and $\mathrm{M}$ gratings have only been taken through the preliminary processes (Figure 6, right) but will be the focus of work during the latter half of 2016. Like GMTIFS, AO is needed for optimal operation at GMT. Nevertheless, the slit width can be widened to $\sim 0.3$ " for use in natural seeing until the AO system is installed, but with degraded throughput and resolution.

Following the grating development, the GMTNIRS team will begin their preliminary design for the rest of the instrument. As noted above, GMTNIRS is designed to be an AO-fed instrument and requires the AO system for optimal performance. The schedule shows that work on GMTNIRS could proceed to a delivery in mid-2022 but AO capabilities are not scheduled for commissioning until mid-2024. Consequently, an early delivery for seeing-limited operation is being discussed within the partnership. 


\subsection{MANIFEST}

The Many Instrument Fiber System (MANIFEST) is a facility fiber utility to feed other instruments, providing access to GMT's full 20' field of view. Because this field of view is very large for an ELT, MANIFEST enables GMT to be highly competitive for $A-\Omega$ survey science without the expense and complexity required to reimage the full focal plane.

MANIFEST can be connected to one or more spectrographs, including GMACS and G-CLEF. Multiplex gains of 5-40 are especially valuable for G-CLEF, which otherwise is a single object spectrograph. Furthermore, MANIFEST can feed both of these instruments simultaneously, adding another multiplexing dimension. MANIFEST can also be expanded in the future to feed a near-IR spectrograph, again separately or simultaneously with the optical instrument feeds. Furthermore, the science projects for those two instruments can be unrelated yet scheduled simultaneously.

Multiplexing gains vary for each spectrograph depending on the available slit length and type of fiber inputs employed (e.g., single object, image slicer, IFU), as well as the demand of the science programs (e.g., bright objects where sky subtraction is not critical can use more object fibers and fewer sky fibers).

The MANIFEST concept uses "Starbugs" - robotic fiber heads that adhere to a glass plate by vacuum forces ${ }^{15}$. This technology has been developed far beyond the concept stage and is being deployed in the TAIPAN ${ }^{7}$ science instrument during the latter half of 2016. Initially delivered with 150 fibers and Starbug positioners, TAIPAN will be upgraded to 300 Starbugs.

Jon Lawrence (AAO) and Matthew Colless (ANU) lead the MANIFEST effort. The key science topics for G-CLEF and GMACS serve as a guide to the science enabled by MANIFEST, but with a multiplexing gain in telescope time. While the TAIPAN project is planning for up to 300 fibers, MANIFEST's much larger physical size allows that count to rise to several thousand.

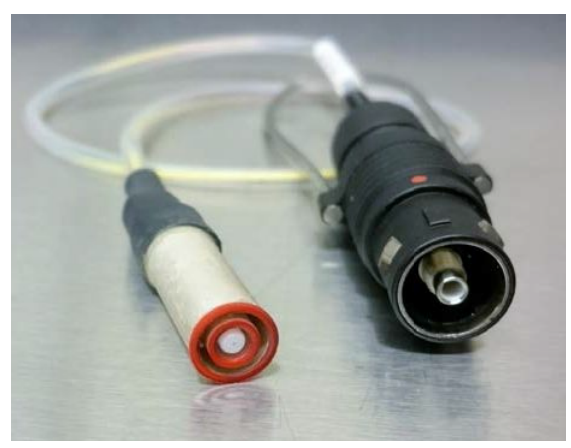

Figure 7. A Starbug for TAIPAN. The left element (white/red) is the Starbug with a science fiber in the center and three metrology fibers in the gap between the two (red) piezo sleeves. The connector on the right mates to a science fiber feeding the spectrograph, the three metrology fibers that are back-lit by LEDs, and power to drive the piezos.

MANIFEST could be delivered early in the GMT staging plan. However, to utilize the full 20' field of view, it requires a wide-field corrector (WFC) to preserve the excellent image quality of the telescope beyond a 10' diameter. An atmospheric dispersion compensator (ADC) is generally needed with a fiber feed system as well. The GMT WFC is integral with the ADC and is scheduled to be delivered 2-3 years after first light. MANIFEST is currently scheduled for delivery in 2023 or earlier. If funding is available and partner interest is strong, MANIFEST can be delivered initially with a smaller 10 arcmin field of view and corresponding ADC to enable MOS and multi-spectrograph capabilities sooner.

\subsection{ComCam}

The Commissioning Camera (ComCam) is a late addition to the GMT instrument suite. Initially specified as an alignment and image quality assessment tool, GMT scientists recognized that it will also be a highly capable optical 
imager with little increase in cost/scope. For a very modest increment in cost, additional filter slots can dramatically increase its scientific potential, especially for narrow-band imaging with GLAO. For broadband imaging, though, other facilities such as LSST can be competitive.

Jeff Crane (Carnegie Observatories) is serving as the PI for ComCam. It is very early in the development process, with a start for conceptual design in Q3 of 2016. Requirements include a pixel scale of 0.06 arcsec/pix and better than 0.10 arcsec image quality to evaluate GLAO performance across its 6'x6' field of view provided by a single 6Kx6K CCD. Suggested science topics include:

- Stellar populations in dwarf and nearby galaxies

- Identification of planetary nebulae (PNe) at $100 \mathrm{Mpc}$ and beyond; distance determinations using the PNLF

- Blind searches for Ly $\alpha$ sources at $\mathrm{z}>6$

ComCam is required to verify the telescope performance at first light. Thus, it must be delivered well before mid-2022 and is scheduled for shipping in mid-2021. This is a 5-year development window, which is short by ELT instrument standards. Fortunately, ComCam is relatively simple, and may be the simplest of all ELT instruments. A notional optical layout is shown in Figure 8, with glasses identified in Table 3.

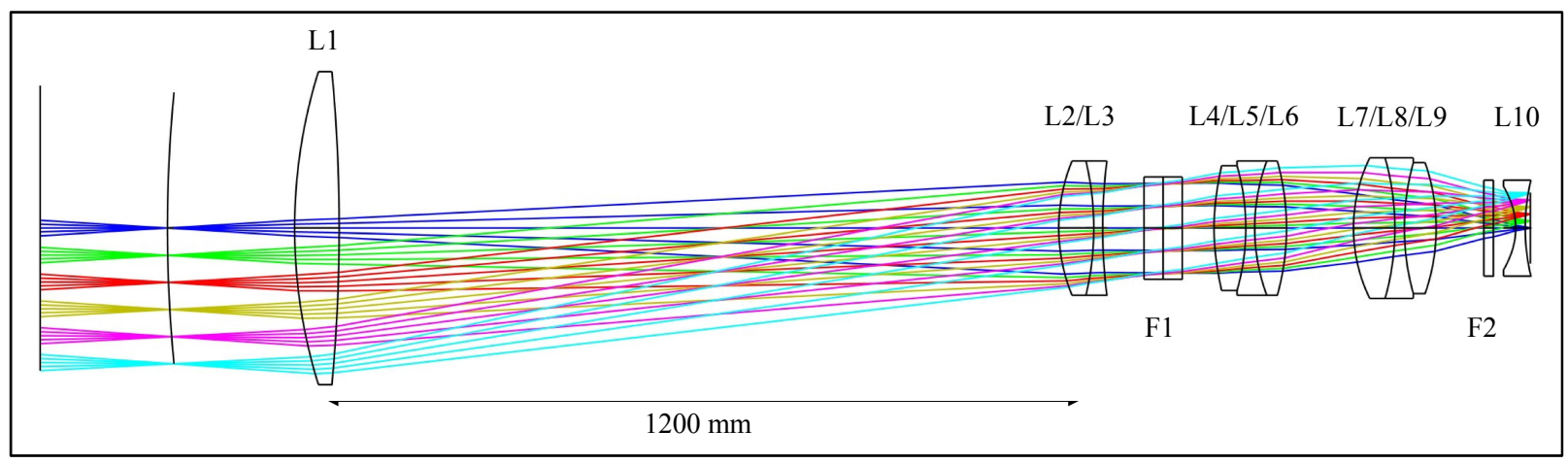

Figure 8. Draft optical layout for Comcam. Elements labeled as F1 and F2 are locations for filters. Narrow-band filters are best located in the nearly collimated zone between elements L3 and L4 whereas broadband filters may be used in the faster converging beam prior to L10.

Table 3. Glass material for the ComCam layout shown above.

\begin{tabular}{|c|c||c|c|}
\hline Lens & Material & Lens & Material \\
\hline L1 & S-FSL5Y & L6 & S-FPL51Y \\
\hline L2 & CaF2 & L7 & CaF2 \\
\hline L3 & BAL35Y & L8 & BAL15Y \\
\hline L4 & CaF2 & L9 & FPL51Y \\
\hline L5 & BAL15Y & L10 & Silica \\
\hline
\end{tabular}

\section{FUTURE INSTRUMENT CONCEPTS}

Each of the ELT projects is thinking about future rounds of instruments because of the lengthy incubation period (10-15 years) and the long time since the first round was originally conceived. GMT further recognizes that its first generation suite was selected in 2012, prior to the concept of staging telescope capabilities over 3-4 years. In that model, adaptive optics is deferred several years and the IR instruments are dragged down the timeline along with the AO, leaving GMT with no IR instrument and no high spatial resolution capability for that period. 
As GMT ramps up the process for identifying and developing future instruments, two first generation candidates that did not survive the down-select will be considered again: NIRMOS ${ }^{16}$, a multiobject, wide-field, near-IR slit spectrograph, and TIGER ${ }^{17}$, a mid-IR, high contrast imager and spectrograph. Both of these have been described in previous conferences. Note that TIGER requires AO.

While the process for advancing new instruments hasn't begun formally, several ideas have emerged to fill those early year capability gaps. Two of these are described below. We expect that an organized development of future instruments, as described by Jacoby ${ }^{2}$, will ramp up before the next conference.

\subsection{SuperFIRE}

Recent thoughts for an early IR instrument at GMT center on a concept derived from FIRE ${ }^{18}$, a popular instrument at Magellan. SuperFIRE ${ }^{19}$ is being led by Rob Simcoe (MIT) and the instrument is described elsewhere in this conference. Since SuperFIRE targets a GMT stage before AO is available, it is necessarily a seeing-limited spectrograph. It offers a spectral resolution of 6000 with continuous wavelength coverage from 0.87-2.50 um split across three spectrograph modules, each with an H2RG detector. As shown in Figure 9, the instrument is small and is neither complex nor expensive for an ELT instrument.

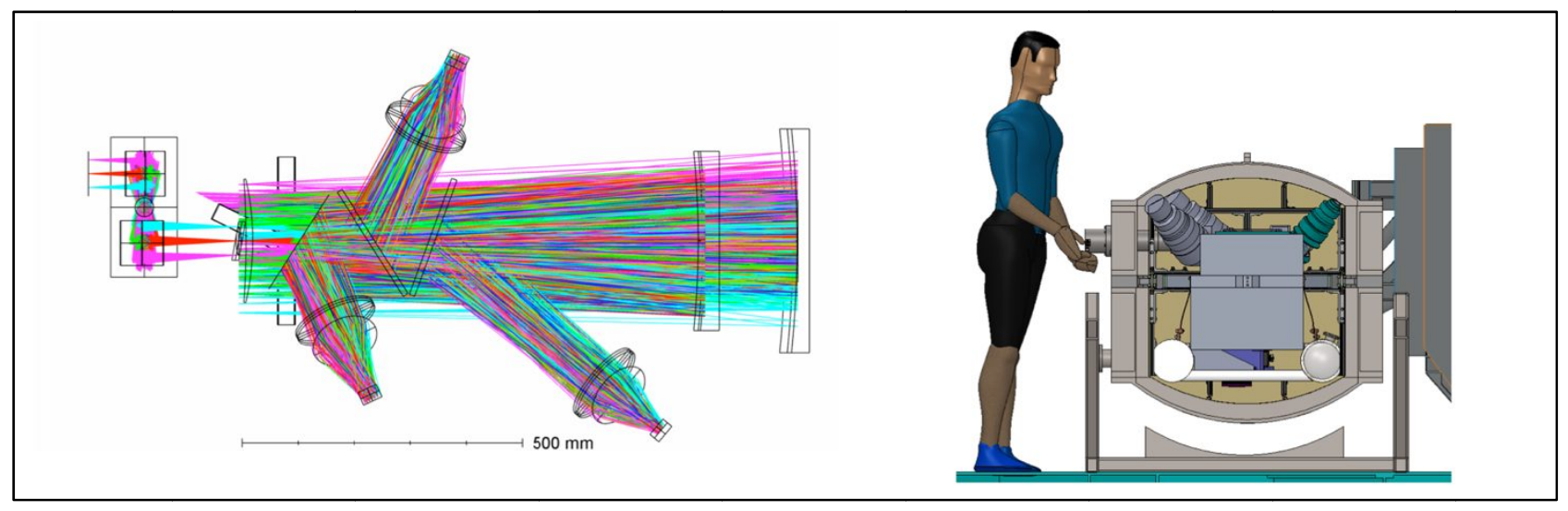

Figure 9. An optical layout for SuperFIRE (left) and a mechanical representation (right) shown for scale.

\subsection{DSSI - A Speckle Imager}

Without AO, GMT will not have a high spatial resolution imaging capability. A very inexpensive approach to fill that gap is with a speckle imager like DSSI ${ }^{20}$ (Differential Speckle Survey Instrument) on Gemini. Elliott Horch (Southern Conn. St. Univ.) and Steve Howell (NASA Ames) have been leading the effort to build, install, and operate speckle imagers at various telescopes, and GMT may be a suitable host.

The two-channel optical system uses EMCCDs to record two color optical images quickly for post-processing (Figure 10). Figure 11 shows an example of a binary star imaged at 562 and $692 \mathrm{~nm}$ with Gemini. 


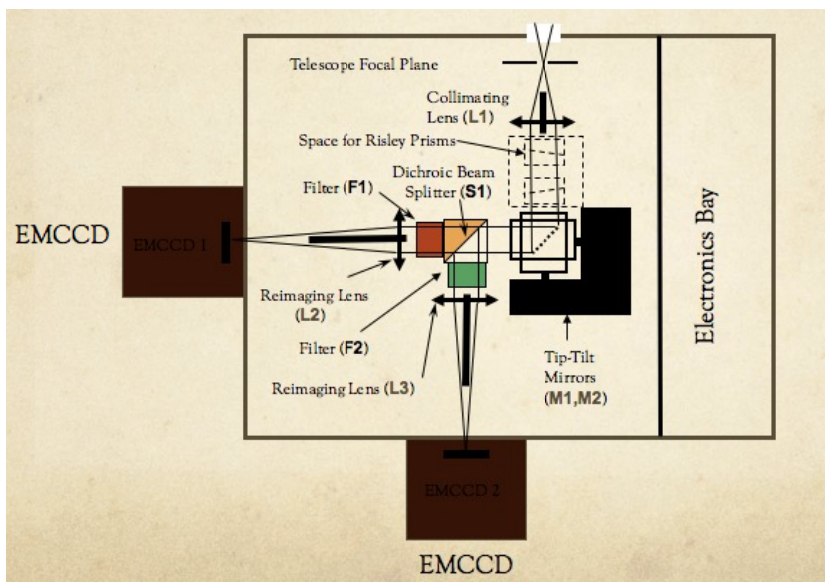

Figure 10. The layout for a speckle camera similar to that deployed at Gemini and the DCT.

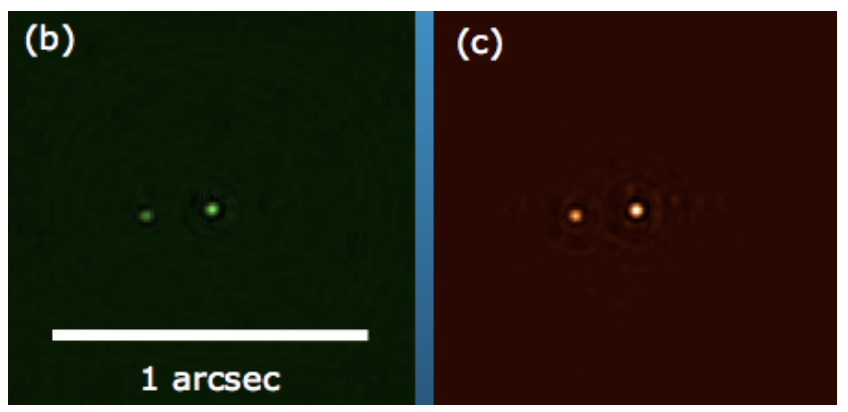

Figure 11. An example of DSSI at Gemini showing a binary star image (after processing). The left image is taken through a filter at $562 \mathrm{~nm}$; the image on the right is taken through a filter at $692 \mathrm{~nm}$.

Because of the simple and pre-existing design of DSSI, and its off-the-shelf component basis, the timeline for fabrication is very short ( $\sim 3$ years) and can easily be ready when needed.

\section{CHALLENGES TO BUILDING ELT INSTRUMENTS}

As a generalization, the instrument development process, from design, fabrication, integration and testing, commissioning, and acceptance, has a reputation for significant overruns in both schedule and cost. There are numerous reasons why overruns happen when building an instrument for facilities at more modest sizes than ELTs, but we focus here on the challenges unique to ELTs. Some of these were alluded to in Section 2. This list is surely incomplete and each ELT instrument team will have further insights.

\subsection{Very Long Development}

As noted earlier, ELT instrumentation can be conceived 10-20 years before it is delivered. The time scale for an ELT instrument and associated facilities tends to be longer than for smaller or existing facilities because (a) the unprecedented capital demands require a lengthy and creative fundraising phase, and (b) the engineering problems include significant research, prototyping, and restarts, and (c) the partnerships are complex and time-varying. A lot can happen in those years:

- Science drivers change as the questions from 10 years ago are partially or fully answered, and new science questions emerge. 
- The telescope design is not stable and so the interfaces and predicted telescope performance changes, demanding redesign of the instruments.

- Key personnel on a team may become unavailable for a variety of reasons (e.g., retirement, illness, change of position), leading to retraining and possibly an irreplaceable talent loss.

- Vendors come and go. A sole-source component may become unobtainable, or a new vendor may appear with a far better product that requires consideration for redesign.

- Technology evolves. For example, detectors and control electronics can improve; new optical glasses are brought to the market. Should you redesign to improve performance?

\subsection{Instruments are Expensive}

- Depending on the corporate model, ELT instruments can exceed $\$ 50 \mathrm{M}$. While that cost is included in the overall project budget, overruns elsewhere in the project will push instrumentation into the future in order to complete the basic facility. In extreme situations, instruments may be canceled.

- Because each instrument is expensive, it is appealing to add as much functionality as possible to each instrument. In doing so, the instrument may become more complex, further increasing cost and delivery time.

- One reason that instruments are expensive is because they are large and heavy, with optics pushing to the maximum dimensions that vendors can produce and where risks are high. Size has other implications, requiring new test facilities and new handling equipment.

\subsection{High Expectations}

- The astronomers who benefit from the existence of ELTs are demanding end users. They expect the data quality from an ELT to vastly surpass the quality from current $8-10 \mathrm{~m}$ facilities in terms of throughput, wavelength coverage, and especially spatial resolution. It is the latter that pushes instrument development into a difficult regime where micron and sub-micron tolerances are the norm, and the parts are large.

\section{COLLABORATIONS}

One approach to mitigating the special challenges of ELT instrument development is for all three projects to work towards solving common problems in a more formal collaborative fashion.

\subsection{Technical Workshops}

A recent example is the ELT Detector Workshop ${ }^{21}$ in October 2015. The three projects were represented by $\sim 30$ attendees from their instrument teams and project offices, along with 16 representatives of the detector industry, both optical and infrared (Figure 12).

Open and frank discussions were held on topics of common interest, such as the availability and performance of detectors and their electronic controller systems, real costs, upcoming developments, and new directions. In return, vendors learned about the short- and long-term demands of the projects. 


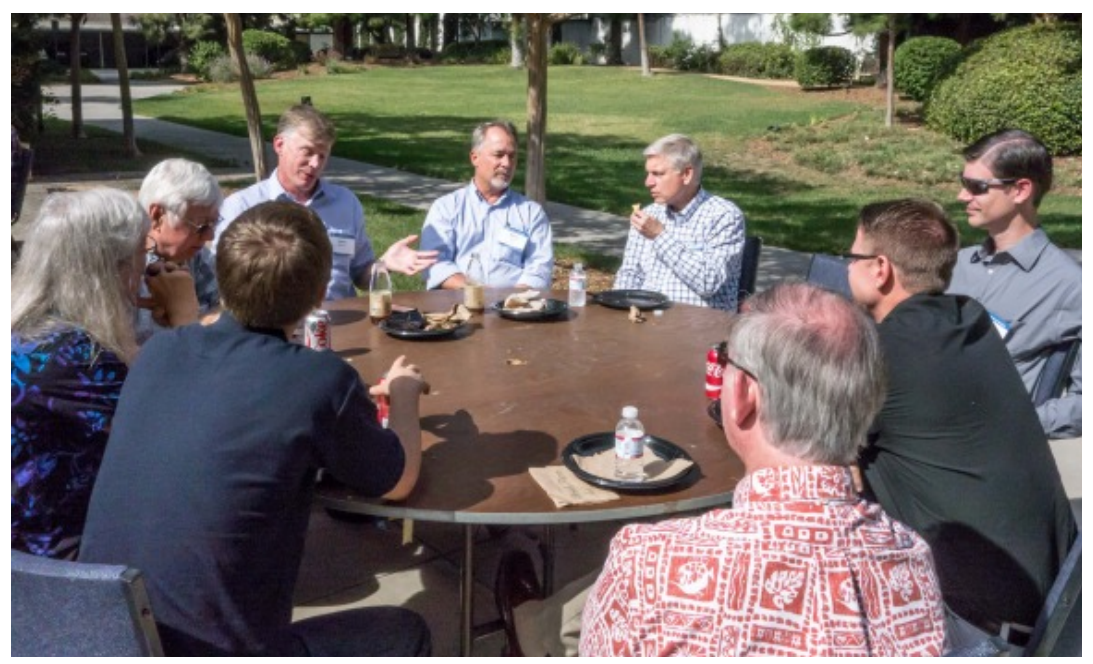

Figure 12. Detector representatives from Raytheon, Teledyne, E2V, STA, and Selex during a lunch break.

Other areas for collaborations exist, for example:

- Gratings, both ruled and VPH

- Optical coatings

- Software, data pipelines, data archives

\subsection{Time Trades}

Another form of collaboration is time trades. As a global community, we should be thinking more about building a system of facilities than building three independent projects. For example, each of the projects is building an AO-fed near-IR IFU spectrograph, and at the same time, there may be instruments that are unique to only one ELT. It is the integrated set of capabilities that astronomers need for their research; maybe we only need one of everything rather than three of everything. The Mauna $\mathrm{Kea}^{22}$ system demonstrates the feasibility of time trades.

\section{ACKNOWLEDGEMENTS}

GHJ wishes to thank the GMTO Board and partners, the project staff, and the instrument development teams for their support, friendship, and sharing their knowledge during my involvement with the project since 2009.

\section{REFERENCES}

[1] Johns, M., et al., GMTO, 2006, http://www.gmto.org/wp-content/uploads/Giant-Magellan-Telescope-ConceptualDesign-Review_GMT-PM-RVW-00146_A.pdf

[2] Jacoby, G. H., et al., "The instrument development and selection process for the Giant Magellan Telescope," Proc SPIE 8446, 84461G (2012).

[3] Szentgyorgyi, A., et al., "The GMT-Consortium Large Earth Finder (G-CLEF): an optical Echelle spectrograph for the Giant Magellan Telescope (GMT)," SPIE 9908-76, (2016).

[4] Sharp, R., et al., "GMTIFS: The Giant Magellan Telescope integral fields spectrograph and imager," Proc SPIE 9908, 9908-72, (2016). 
[5] DePoy, D., et al., "The Giant Magellan Telescope multi-object astronomical and cosmological spectrograph (GMACS)," Proc SPIE 9908, 9908-79 (2016).

[6] Jaffe, D., et al., "GMTNIRS: progress toward the Giant Magellan Telescope near-infrared spectrograph," Proc SPIE 9908, 9908-75 (2016).

[7] Lawrence, J., et al., "The MANIFEST prototyping design study,” Proc SPIE 9908, 9908-358 (2016).

[8] McCarthy, P., et al., "Overview and status of the Giant Magellan Telescope Project," Proc SPIE 9906, 9906-37 (2016).

[9] Abbot, B. P. et al., "GW151226: Observation of Gravitational Waves from a 22-Solar-Mass Binary Black Hole Coalescence,” Phys. Rev. Lett. 116, 241103-1:14 (2016).

[10]McCarthy, P., (ed.), GMTO, 2012, "Giant Magellan Telescope Scientific Promise and Opportunities," http://www.gmto.org/Resources/GMT-SCI-REF-00482_2_GMT_Science_Book.pdf

[11] Ladd, J. A., et al., "Site and enclosure CFD modeling and analysis for the Giant Magellan Telescope," Proc SPIE 9911, 9911-42 (2016).

[12]Danks, R., et al., "Initial computational fluid dynamics modeling of the Giant Magellan Telescope site and enclosure,” Proc SPIE 9911, 9911-41 (2016).

[13]P. McGregor, J. Hart, P. Conroy, L. Pfitzner, G. Bloxham, D. Jones, M. Downing, M. Dawson, P. Young, M. Jarnyk and J. van Harmelen, "Gemini near-infrared integral field spectrograph (NIFS)," Proc SPIE 4841, 178 (2002).

[14] Mace, G., et al., “300 nights of science with IGRINS at McDonald Observatory,” Proc SPIE 9908, 9908-11 (2016).

[15] Lawrence, J., et al., "The MANIFEST fibre positioning system for the Giant Magellan Telescope," Proc SPIE 9147, 914794 (2014).

[16]Fabricant, D., et al., "NIRMOS: a wide-field near-infrared spectrograph for the Giant Magellan Telescope," Proc SPIE 8446, 844610 (2012).

[17] Hinz, P., et al., TIGER: a high contrast infrared imager for the Giant Magellan Telescope," Proc SPIE 8446, 84461P (2012).

[18] Simcoe, R. A., et al., "FIRE: A Facility Class Near-Infrared Echelle Spectrometer for the Magellan Telescopes," PASP $125,270-286(2013)$.

[19] Simcoe, R., et al., “A Concept for Seeing-Limited Near-IR Spectroscopy on the Giant Magellan Telescope,” Proc SPIE 9908, 9908-382 (2016).

[20] Horch, E. P., et al., "Observations of Binary Stars with the Differential Speckle Survey Instrument. I. Instrument Description and First Results", AJ 137, 5057 (2009).

[21] Jacoby, G. H., et al., GMTO, 2015, http://www.gmto.org/elt-detector-workshop/

[22] Hand, E., "Astronomers set up telescope timeshare," Nature, 2012, http://www.nature.com/news/astronomers-setup-telescope-timeshare-1.11725 